Abstract P240 Table 1 Pneumothorax management in cystic fibrosis patients

\begin{tabular}{llrlll}
\hline Treatment & $\begin{array}{l}\text { Episodes of } \\
\text { pneumothorax }\end{array}$ & $\begin{array}{l}\text { Resolution } \\
\text { rate }\end{array}$ & $\begin{array}{l}\text { Second line } \\
\text { treatment }\end{array}$ & Complications & $\begin{array}{l}\text { Late } \\
\text { recurrence }\end{array}$ \\
\hline Observation & 13 & $8(62 \%)$ & 4 (chest drain) & 0 & $4(40 \%)$ \\
$\begin{array}{l}\text { Chest drain } \\
\text { Chemical (talc) }\end{array}$ & 18 & $13(59 \%)$ & 8 (surgery) & 4 deaths & $8(62 \%)$ \\
pleurodesis & & $6(33 \%)$ & 2 (surgery) & 1 death & $4(57 \%)$ \\
\begin{tabular}{l} 
Surgery \\
\hline
\end{tabular} & 10 & $5(50 \%)$ & - & 5 deaths & $1(20 \%)$ \\
\hline
\end{tabular}

Conclusion Thus, we have confirmed that spontaneous pneumothorax is a poor prognostic factor in CF, with $57 \%$ of patients experiencing a recurrent pneumothorax within the first year and poor one and 2-year survival rates. Surgical treatment was associated with a lower risk of recurrence, but at the expense of an increase in mortality. Randomised controlled trials of treatment options and the formulation of guidelines are required to determine the timing and optimal management to prevent recurrent pneumothorax in CF patients.

\section{P241 ACCUCHEK (TM) MEASUREMENT OF BLOOD GLUCOSE CORRELATES WITH TRUE LAB GLUCOSE MEASUREMENT IN CHILDREN SCREENED FOR CYSTIC FIBROSIS RELATED DIABETES}

doi:10.1136/thoraxjnl-2011-201054c.241

A J Fall, A Mallinson, A Mill, D S Urquhart. Royal Hospital For Sick Children, Edinburgh, $U K$

Background Screening for cystic fibrosis related diabetes (CFRD) is recommended as part of the cystic fibrosis annual review. For children over 10 an oral glucose tolerance test (OGTT) is recommended, but this is time consuming and adds to the burden of assessments and investigations of the annual review. As part of a proposed wider pragmatic study to evaluate the feasibility of home OGTT testing in this patient group, we assessed the level of agreement between blood glucose concentration measured by near-patient testing using the Accucheck glucometer and that measured in the laboratory using standard methods (True Lab Glucose-TLG).

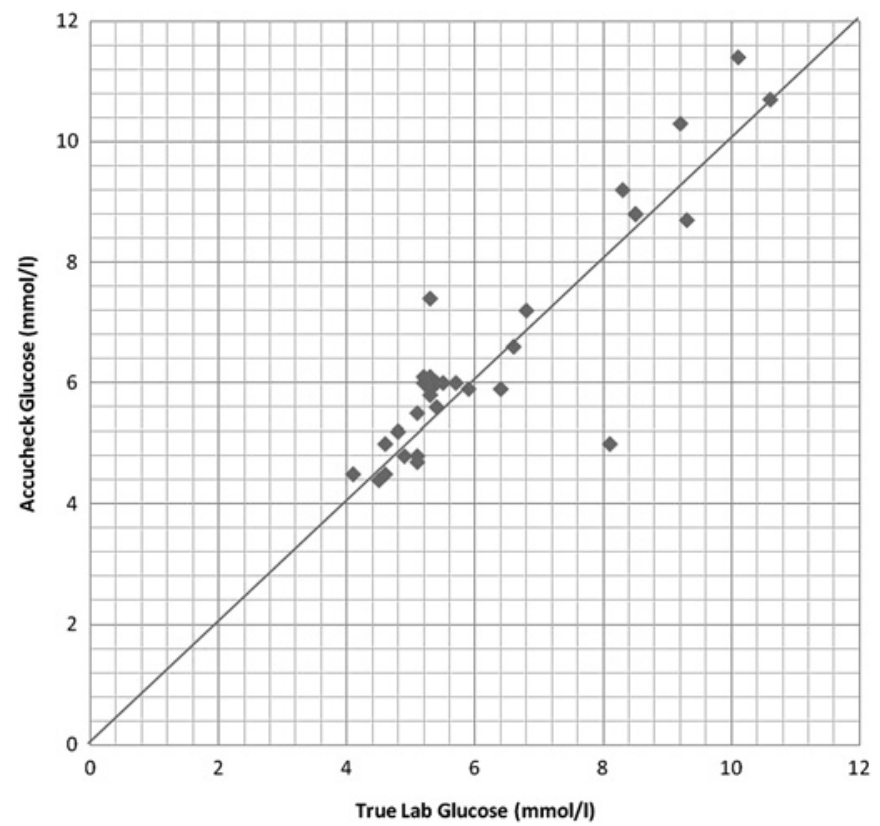

Abstract P241 Figure 1
Methods 26 children with CF aged 1 yr-16 yrs undergoing annual review were studied. Blood taken at the time of venepuncture was tested for glucose concentration using an Accucheck glucometer, and results compared with TLG. For children under 10 a single, random blood glucose was tested using each method. For those aged 10 or over samples taken after an overnight fast, and $2 \mathrm{~h}$ after a standard OGTT oral glucose load were tested using each method. Bland and Altman statistics were used to assess limits of agreement between methods of glucose measurement.

Results 31 pairs of glucose measurements were obtained. TLG measurements ranged from 4.1 to $10.6 \mathrm{mmol} / \mathrm{l}$. Abstract P241 Figure 1 shows the Accucheck glucose measurement plotted against the TLG for each sample, and the line of equality. The agreement between Accucheck and TLG was investigated using the method of Bland and Altman and showed that the Accucheck gave results a mean (SD) of $0.26(0.84) \mathrm{mmol} / 1$ higher than values obtained on TLG.

Conclusions There is a clinically acceptable agreement between blood glucose measured by Accucheck compared with TLG. Just one child with a TLG result that might have prompted further investigation could have been missed, had Accucheck been the sole method used. This suggests that blood glucose measurement in the context of OGTT screening for CFRD could perhaps be performed using near-patient testing in an out of hospital setting (eg, by parents at home) without compromising test validity. Further research is needed to establish if this is the case.

\section{P242 BONE DENSITY AND TESTOSTERONE LEVELS IN MALE CYSTIC FIBROSIS PATIENTS}

doi:10.1136/thoraxjnl-2011-201054c.242

B Marson, C Valsalakumari, L Speight, J Platts, L George, R I Ketchell, J Duckers. University Hospital Llandough, Cardiff, UK

Background Cystic Fibrosis (CF) is the commonest inherited life threatening condition in the UK and is a multisystem disease. CF related low bone mineral density (BMD) is defined as a $Z$ score 2 SDs below the age and gender matched mean reference value and has been reported in $13 \%$ of CF patients. ${ }^{1}$ Its aetiology is multifactorial with hypogonadism being a potential factor. CF Trust guidelines $^{2}$ suggest BMD should be measured and addressed regularly and testosterone levels measured annually in adult males.

Aim To assess the prevalence of low BMD and testosterone in male CF patients attending the All Wales CF Centre.

Methods Annual review records (2008-2009) of BMD derived from DEXA scans and testosterone levels were reviewed retrospectively.

Results 65 male patients (age 18-51 years) were included of whom 60 had a recorded testosterone and BMD. 12 patients $(20 \%)$ had CF related low $\mathrm{BMD}$ (at hip or lumbar spine) of whom three had a low testosterone (<8.0 nmol/l). $12(20 \%)$ patients had low testosterone levels. Of these twelve, three had low leutinising hormone (LH) and nine normal LH and all had normal levels of follicle stimulating hormone.

Discussion CF related low BMD occurs commonly in men with CF with a quarter of those with low BMD also having low testosterone levels. Low testosterone levels per se also occur frequently in this population and recent CF Trust guidelines ${ }^{2}$ have suggested an endocrinology opinion should be sought where testosterone is found to be low.

\section{REFERENCES}

1. Elkin SJ, et al. Osteoporosis Int 2001

2. UK Cystic Fibrosis Bone Mineralisation Working Group. 2007. 